\title{
Design Optimization of PMU Anti-Aliasing Filters using Taguchi Method
}

\author{
Fatma Zohra DEKHANDJI \\ Laboratory signals and systems, Institute of electrical and electronic engineering; University \\ M'hamed Bougara de Boumerdes; Avenue de l'indépendance, 35000. ALGERIA \\ fzdekhandji@univ-boumerdes.dz
}

\begin{abstract}
A Phasor Measurement Unit (PMU) is a monitoring device, which serves in checking the power system condition by measuring voltage and current phasors along with frequency at a particular node. The basic structure of PMU consists of Synchronization Unit, Measurement Unit and Data Transmission Unit. The Measurement Unit has three components: Anti-aliasing filters, Analog-to-Digital Converter and Phasor measurement Unit/ Processor. An anti-aliasing filter ensures that all the analog signals have the same phase shift and attenuation thus assuring that the phase angle differences and relative magnitudes of the different signals are unchanged. Anti-aliasing filters made up of an analog front end and a digital decimation filter are far more stable as far as aging and temperature variations are concerned. IEEE C37.118 standard stipulates that it is mandatory to use the filter for avoiding any aliasing errors. Out of various analog filters, the Butterworth has been preferred due to its flat response in pass-band as compared to other filters. In this work, it is attempted to design anti-aliasing filters to be used in PMUs. The design problem is formulated as an optimization task that is solved using the Taguchi method. The results show better performance in terms characteristics compared to the conventional filters. The designed filters may be employed as building blocks in modern PMUs.
\end{abstract}

Keywords: Anti-Aliasing filters, optimization, Taguchi method, PMU.

\section{INTRODUCTION}

Filtering in its raw definition is any of the various mechanical, physical, electrical or biological operations that separate solids from fluids liquids or gases by adding a medium through which only the fluid can pass. The fluid that passes through is called the filtrate. In circuit theory, a filter is a circuit capable of passing or amplifying certain frequencies while attenuating other frequencies. Thus, a filter can extract important frequencies from signals that also contain undesirable or irrelevant frequency. Filters can be placed in one of two classes: analog or digital. Analog filters can be passive where circuits contain passive elements like capacitors, inductors and resistors or active in which the circuits use an operational amplifier (op amp) as an active device in combination with some resistors and capacitors. Digital filters are implemented in software using a digital computer or special purpose digital hardware $[2,13,14]$.

Ideally, filters are classified into four basic filter types: low-pass, high-pass, band-pass and all-pass. They are classified according to theirs magnitude and phase responses [115]. Some states of art practical approximations have been proposed [2-3]. The first filter approximation is the Butterworth or maximally-flat response. It exhibits a nearly flat pass-band with no ripple and the roll off is smooth $[3,7,14]$. Another approximation to the ideal filter is the Chebychev or equi-ripple response. This filter has ripple in the pass-band amplitude response $[3,7,14]$. A third filter is the Bessel filter characterized by a linear phase response with respect to frequency $[3,7,14]$. Due to the presence of the denominator of the transfer function, the stability condition of the filter should be taken into account in the optimal design [16], [17-22], [23-26], [2733], [34], [35], [36], resulting in a constrained optimization problem. Several sufficient conditions [17-19], [21], [25], [27], [33] have been established for the parameterization that represents the filter's denominator by a single polynomial. The triangle-based stability conditions [16] are necessary and sufficient and have been incorporated into several design procedures [26], [28], [29] that formularize the filter's denominator by cascaded second-order sections (SOSs). In [28], variable transformation is used to convert the finite stability region into the entire coefficient space, such that the original constrained design problem becomes an 
unconstrained one in the transformed space. However, the transformation increases the nonlinearity of the objective function, which makes it hard to find good (global optimum) solutions in general. In [29], a perturbed stability triangle is proposed to guarantee the SOS to have its zeroes inside a circle of given radius. It is combined with the GaussNewton strategy, resulting in an improved design. In [30], the conditions presented for the SOS with zeroes inside a circle of given radius enclose a triangular stability domain and can be easily incorporated into any constrained optimization formulations based on the SOS parameterization. A method that divides the overall design of an IIR filter into successive designs of its second-order sections is presented in [35], where one section is first designed, and then, another section is appended until all sections are designed.

The goal of this paper is to employ the Taguchi optimization technique to design anti-aliasing filters that can be embedded within a Phasor Measurement Unit (PMU). The design criteria are to meet some set properties that are: maximum passband flatness; an acceptable passband-tostopband transition and to have a linear phase response. The designed filters are compared to the state of art designs such as the Butterworth, Chebychev and Bessel types. It is found that the proposed approach produces filters with better properties as the state of art prototypes meet a single objective at a time from the set of objectives in the optimization task. The designed filters exhibit a compromise between these properties and hence are very useful for practical PMUs.

\section{PROBLEM FORMULATION}

Practically, an active Filter transfer function can be written as

$$
H(s)=\frac{A_{0}}{\prod_{i}\left(1+a_{i} s+b_{i} s^{2}\right)}
$$

Where $i$ denotes the second order section. $\mathrm{A}_{0}$ is the OPAMP gain. $a_{i}$ and $b_{i}$ denote the coefficients of the second order section of the overall filters and $s$ is the Laplace transform operator.

The transfer function in (1) evaluated on the $j \omega$ axis is a complex quantity. Hence, it has amplitude (called the magnitude response) and an argument (called the phase response). Both quantities are functions of the frequency $\omega$. Ideally, the magnitude and phase responses of a low-pass filter can be optimized to satisfy the following three criteria:

1) A maximum passband flatness,

2) An immediate passband-to-stopband transition,

3) A linear phase response.

A typical practical lowpass filter magnitude response is shown in Fig.1.a. Ideally, the designed filter must have a magnitude response like the one shown in Fig. 1.b.

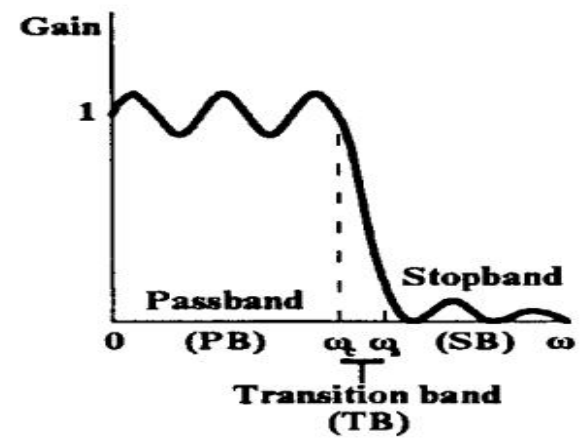

(a)

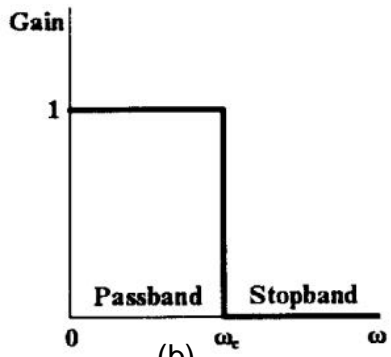

(b)

Fig. 1 Low-Pass filter magnitude response:

(a) Practical filters. (b) Ideal Filters

It is clear that to achieve this (although not practically realizable), the designer must make the passband as flat as possible and the transition band as small as possible.

To satisfy the two set objectives, the following sub-objective functions are used:

- The maximum flatness is ensured if the least squares error between the designed filter and the flat response in the passband (set as 1) is minimized. This is given mathematically as:

$$
f_{1}=\sum_{n}\left(\operatorname{Gain}_{p}-1\right)^{2}
$$

The smallest possible transition band is obtained if the difference between the ending frequency of the passband and the starting frequency of the stopband is minimized. This is formulated mathematically as:

$$
f_{2}=\left|\omega_{s}-\omega_{p}\right|
$$

To satisfy the three criteria, a weighted function is used in the optimization task written as: 


$$
f_{o b j}=\alpha f_{1}+\beta f_{2}
$$

To formulate the design problem as an optimization task, the following steps apply:

1. Define filter specifications: The order, frequency of operation, cutoff frequency, bandpass maximum ripple, transition bandwidth, bandstop maximum ripple and the OPAMP gain.

2. Decide on the sectioning pattern of the filter (depending on whether the order is odd or even.)

3. Set the limits of the coefficients in each section.

4. Apply the Taguchi method using the function in (4).

\section{THE TAGUCHI OPTIMIZATION METHOD}

Thanks to the rapid development of computer technology, many optimization techniques such as genetic algorithm (GA), particle swarm optimization (PSO), simulated annealing (SA), artificial neural network (ANN), and gradient-based techniques have been implemented by computer codes.

Taguchi's method was developed based on the concept of the orthogonal array $(O A)$, which can effectively reduce the number of tests required in a design process [37]. It provides an efficient way to choose the design parameters in an optimization procedure.

Before presenting the Taguchi procedure, it is worth understanding what OAs are and how are they generated [37]. Let $S$ be a set of $s$ symbols or levels (the simplest symbols are integers $1,2,3 \ldots$.). A matrix $A$ of $N$ rows and $k$ columns with entries from $S$ is said to be an OA with s levels and strength $t(0<t<k$ ) if in every $N \times t$ subarray of $A$, each $t$-tuple based on $S$ appears exactly the same times as a row. The notation $\mathrm{OA}(N, k, s, t)$ is used to represent an OA.

\section{Initialization procedure}

The optimization procedure starts with the problem initialization, which includes the selection of a proper $O A$ and the design of a suitable fitness function. The selection of an $\mathrm{OA}(N, k, s, t)$ mainly depends on the number of optimization parameters. In general, to characterize the nonlinear effect, three levels $(s=3)$ are found sufficient for each input parameter. Usually, an OA with a strength of $2(t=2)$ is efficient for most problems because it results in a small number of rows in the array.

\section{Design of input parameters}

The input parameters need to be selected to conduct the experiments. When the $\mathrm{OA}$ is used, the corresponding numerical values for the three levels of each input parameter should be determined.

In the first iteration, the value for level 2 is selected at the center of the optimization range. Values of levels 1 and 3 are calculated by subtracting/adding the value of level 2 with a variable called level difference (LD). The level difference in the first iteration (LD1) is determined by the following equation:

$$
L D_{1}=\frac{\text { Max }- \text { Min }}{\text { Number of levels }+1}
$$

Where Max is the upper bound of the optimization range and $\mathrm{Min}$ is the lower bound of the optimization range.

\section{Conduct Experiments and Build a Response Table}

After determining the input parameters, the fitness function for each experiment can be calculated. These results are then used to build a response table for the first iteration by averaging the fitness values for each parameter $n$ and each level $m$ using the following equation:

$$
F_{a v}=\frac{s}{N} \sum_{i, O A(i, n)=m} f_{i}
$$

Identify Optimal Level Values and Conduct Confirmation Experiment

Finding the largest fitness value ratio in each column can identify the optimal level for that parameter. When the optimal levels are identified, a confirmation experiment is performed using the combination of the optimal levels identified in the response table. This confirmation test is not repetitious because the OA-based experiment is a fractional factorial experiment, and the optimal combination may not be included in the experiment table. The fitness value obtained from the optimal combination is regarded as the fitness value of the current iteration.

\section{Reduce the Optimization Range}

If the results of the current iteration do not meet the termination criteria, the process is repeated in the next iteration. The optimal level values of the current iteration are used as central values (values of level 2) for the next iteration. To reduce the optimization range for a converged result, the $L D i$ is 
multiplied with a reduced rate $(r r)$ to obtain $\mathrm{LD}_{\mathrm{i}+1}$ for the $(i+1)^{\mathrm{th}}$ iteration:

$$
L D_{i+1}=r r \times L D_{i}=R R(i) \times L D_{i}
$$

Where $R R(i)$ is called reduced function. When a constant $\mathrm{rr}$ is used, $R R(I)=r r^{\prime}$. The value of $r r$ can be set between 0.5 and 1 depending on the problem. The larger $r r$ is, the slower the convergence rate.

If $L D_{i}$ is a large value, and the central level value is located near the upper bound or lower bound of the optimization range, the corresponding value of level 1 or 3 may reside outside the optimization range. Therefore, a process of checking the level values is necessary to guarantee that all level values are located within the optimization range. If an excessive situation happens, reassigning the level value for the parameter will be performed. A simple way is to use the boundary values directly.

\section{Check the Termination Criteria}

When the number of iterations is large, the level difference of each element becomes small from equation (7). Hence, the level values are close to each other and the fitness value of the next iteration is close to the fitness value of the current iteration. The following equation may be used as a termination criterion for the optimization procedure:

$$
\frac{L D_{i}}{L D_{1}}<\text { converged value }
$$

Usually, the converged value can be set between 0.001 and 0.01 depending on the problem. The iterative optimization process will be terminated if the design goal is achieved or if equation (8) is satisfied.

\section{RESULTS AND DISCUSSIONS}

From the previous discussions, the design of the analog anti-aliasing filter aims at finding the coefficients $a_{i}$ 's and $b_{i}$ 's in equation (1) so that:

- The magnitude response in the passband is as flat as possible;

- The transition band is as small as possible; and

- The phase response is linear in the passband.

A $6^{\text {th }}$ order filter is to be designed. For simplicity, we consider $A_{0}$ to be 1 . A comparison of the Butterworth, Chebychev and Bessel state of art filters in terms of magnitude and phase responses is shown in Fig. 2.

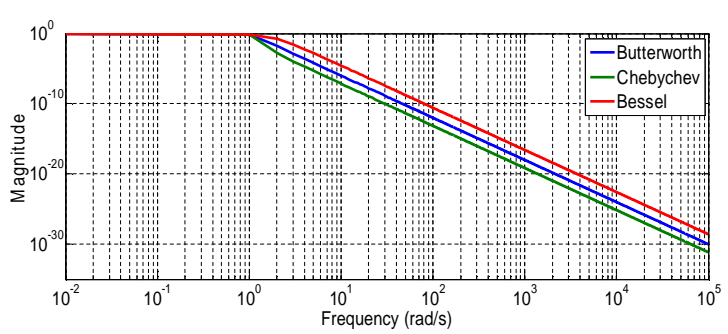

(a)

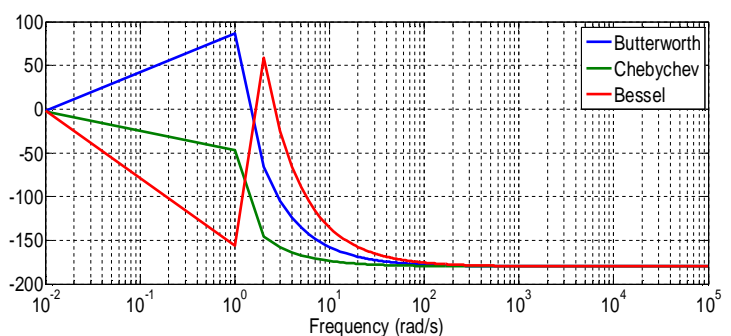

(b)

Fig. 2 Magnitude and phase responses of benchmark filters: (a) Magnitude response;

(b) Phase response

To test the designed filter, a synthetic test signal has been applied to it and Fig. 3 shows both the input and filtered waveforms. It can be seen that the designed filter succeeded in filtering properly the waveform and a purely sinusoidal waveform has been produced.

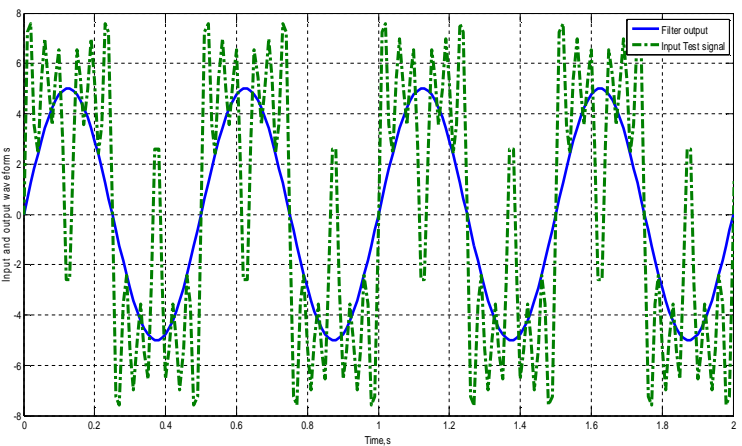

Fig. 3 The designed filter under test signal

\section{CONCLUSION}

The application of the Taguchi optimization method to design an anti-aliasing filter has been dealt with in this paper. The objectives of the filter design were to match a desired magnitude response while having a linear phase. The Taguchi optimization method has succeeded in reaching the optimal design in terms of the desired requirements by achieving a compromise between them. The optimized filter has been tested and it showed good performance with required characteristics which makes it of practical use in Phasor Measurement Units. 


\section{References}

[1] Kerry lacanette, "A Basic introduction to FiltersActive, Passive and Switched-Capacitor", National semiconductor Application Note 779 April 1996.

[2] A. Antoniou,"Digital Filters: Analysis, Design, and Applications", NY: McGraw-Hill, 1993 New York.

[3] Larry D.Paarmann" Design And Analysis Of Analog Filters,A Signal Processing Perspective". Kluwer Academic Publishers, 2001.

[4] Huelsman, L.P. (1993). Active and Passive Analog Filter Design: An Introduction, McGrawHill, New York.

[5] James E. Brittain, "The Introduction of the Loading Coil: George A. Campbell and Michael I. Pupin", Technology and Culture, Vol. 11, No. 1 (Jan., 1970), pp. 36-57, The Johns

[6] Piloty, Hans (1957), "Cauer, Wilhelm", Neue Deutsche Biographie (NDB) (in German), 3, Berlin: Duncker \& Humblot, pp. 179-180.

[7] Haykin, S. (1989). Modern Filters, Macmillan, New York.

[8] Thomasena Brenna"Hand book of Electric current and Electronics", leo Goldberg.revised Edition 2016

[9] S. Winder, «Analog and digital filter design », $2^{\text {nd }}$ edition, Elsevier, 2002.

[10] S. A. PACTITIS" ACTIVE FILTERS Theory and Design".

[11] S. Franko, Design with Operational Amplifiers and Analog Integrated Circuits, McGraw-Hill, 1988.

[12]Sergio Franco, "Design with Operational Amplifier and Analog Integrated Circuits", third edition, McGraw Hill 2003.

[13] Les Thede "Practical Analog and Digital Filter Design", Artech House, Inc, 2004

[14] Jim Karki, "Active Low-Pass Filter Design", Texas Instruments, 2000.

[15] Abdelmadjid RECIOUI, Fatma Zohra HAMRIOUI, Kenza BENAMROUCHE, Oussama MERABET, "Analog Filter Design: A Succinct Investigation", Algerian Journal of Signals and Systems, Vol.3, Issue 4, December 2018. pp:174189.

[16] Antoniou, A.: Digital Filters: Analysis and Design, 2nd ed. New York. McGraw Hill (1993).

[17] Antoniou A.: Digital signal processing: signals, systems and filters, McGraw Hill (2006).

[18] Dumitrescu, B. and Niemisto, R.: Multistage IIR filter design using convex stability domains defined by positive realness. IEEE Trans. Signal Process. 52 (4), 962-974 (2004).

[19] C. Y.-F. Ho, B. W.-K. Ling, Z.-W. Chi, M. Shikh-Bahaei, Y.-Q. Liu, and K.-L. Teo: Design of near-allpass strictly stable minimal-phase real valued rational IIR filters. IEEE Trans. Circuits Syst. II, Exp. Briefs 55 (8), 781-785 (2008).

[20] A. Jiang and H. K. Kwan: IIR digital filter design with new stability constraint based on argument principle. IEEE Trans. Circuits Syst. I, Reg. Papers 56 (3), 583-593 (2009).

[22] A. Jiang and H. K. Kwan: Minimax design of IIR digital filters using iterative SOCP. IEEE Trans.
Circuits Syst. I, Reg. Papers 57 (6), 1326-1337 (2010).

[23] A. Jiang and H. K. Kwan: Minimax design of IIR digital filters using SDP relaxation techniques. IEEE Trans. Circuits Syst. I, Reg. Papers 57(2), 378-390 (2010).

[24] X. P. Lai and Z. P. Lin: Minimax design of IIR digital filters using a sequential constrained leastsquares method. IEEE Trans. Signal Process. 58(7),3901-3906 (2010).

[25] M. C. Lang: Least-squares design of IIR filters with prescribed magnitude and phase responses and a pole radius constraint. IEEE Trans. Signal Process. 48(11),3109-3121(2000).

[26] W. S. Lu: An argument-principle based stability criterion and application to the design of IIR digital filters. Proc. IEEE Int. Symp. Circuits Syst., Island of Kos, Greece, 4431-4434 (2006).

[27] W. S. Lu: Design of stable minimax IIR digital filters using semidefinite programming. Proc. IEEE Int. Symp. Circuits Syst., Geneva, Switzerland 1, 355-358 (2000).

[28] W. S. Lu: Design of recursive digital filters with prescribed stability margin: A parameterization approach. IEEE Trans. Circuits Syst. II, Analog Digit. Signal Process 45(9), 12891298 (1998).

[29] W. S. Lu and T. Hinamoto: Optimal design of IIR digital filters with robust stability using conic quadratic-programming updates. IEEE Trans. Signal Process. 51(6), 1581-1592 (2003).

[30] M. C. Lang: Constrained design of digital filters with arbitrary magnitude and phase responses. Ph.D. dissertation, Vienna Univ. Technol., Vienna,Austria (1999).

[31] W. S. Lu, S. C. Pei, and C. C. Tseng: A weighted least-squares method for the design of stable 1-D and 2-D IIR digital filters. IEEE Trans. Signal Process. 46(1), 1-10 (1998).

[32] O. I. Omoifo and T. Hinamoto: Optimal design of stable recursive digital filters using unconstrained optimization methods. Proc. Int. Midwest Symp. Circuits Syst., Hiroshima, Japan 2, II-49-II-52 (2004).

[33] S. Saab, W. S. Lu, and A. Antoniou: Design and implementation of lowpower IIR digital filter systems. Proc. IEEE Int. Symp. Circuits Syst., Orlando, FL3, 391-394 (1999).

[34] C. K. Sanathanan and J. Koerner: Transfer function synthesis as a ratio of two complex polynomials. IEEE Trans. Autom. Control AC-8(1), 56-58 (1963).

[35] C. C. Tseng and S. L. Lee: Minimax design of stable IIR digital filter with prescribed magnitude and phase responses. IEEE Trans. Circuits Syst. I, Fundam. Theory Appl. 49(4), 547-551 (2002).

[36] Weng Wei-Chung, Yang Fan and Elsherbeni Atef (2007), Electromagnetics and Antenna Optimization Using Taguchi's Method, Morgan and Claypool Publishers.

[37] A. Ouadi, H. Bentarzi and A. Recioui, "Optimal Multiobjective Design of Digital Filters using Taguchi Optimization tecinique", Journal of Electrical Engineering, Vol.65, Issue 1, 2014. pp:21-29. 